\title{
Pervasive effects of Wolbachia on host activity
}

Michael T.J. Hague ${ }^{1, *}$, H. Arthur Woods ${ }^{1}$, and Brandon S. Cooper ${ }^{1}$

${ }^{1}$ Division of Biological Sciences

University of Montana

32 Campus Dr.

Missoula, MT 59812

\section{*Corresponding Author}

Michael T.J. Hague

Division of Biological Sciences, University of Montana

32 Campus Dr. HS 104, Missoula, MT 59812

(406) 243-5122

michael.hague@mso.umt.edu 


\section{ABSTRACT}

Heritable symbionts have diverse effects on the physiology, reproduction, and fitness of

3 their hosts. Maternally transmitted Wolbachia are one of the most common endosymbionts in

4 nature, infecting about half of all insect species. We test the hypothesis that Wolbachia alter host

5 behavior by assessing the effects of 14 different Wolbachia strains on the locomotor activity of

6 nine Drosophila host species. We find that Wolbachia alter the activity of six different host

7 genotypes, including all hosts in our assay infected with $w$ Ri-like Wolbachia strains (wRi, wSuz,

8 wAur), which have rapidly spread among Drosophila species in only the last 13,000 years. While

9 Wolbachia effects on host activity were common, the direction of these effects varied

10 unpredictability and sometimes depended on host sex. We hypothesize that the prominent effects

11 of wRi-like Wolbachia may be explained by patterns of Wolbachia titer and localization within

12 host somatic tissues, particularly in the central nervous system. Our findings support the view

13 that Wolbachia have wide-ranging effects on host behavior. The fitness consequences of these

14 behavioral modifications are important for understanding the evolution of host-symbiont

15 interactions, including how Wolbachia spread within host populations. 


\section{INTRODUCTION}

Insects harbor microorganisms that have wide-ranging effects on their performance and fitness [1-3], including manipulations to reproduction [4-7], provisioning of nutrients $[1,8,9]$, modifications of thermotolerance [10,11], and defense against pathogens [12-15]. Microbes may also alter host behavior [16-21]. In extreme instances, parasitic microbes can induce behaviors that increase the likelihood of transmission - for example, by directing hosts to habitats that promote transmission [22-28]. Infected hosts may also change their own behavior as an immune

23 strategy against infection, including seeking warm temperatures to induce a "behavioral fever"

$24[29,30]$ or reducing activity and increasing sleep time [19,31-34]. Such behavioral modifications

25 have important implications for microbe spread and host fitness.

Maternally transmitted Wolbachia are the most common endosymbionts in nature,

27 infecting many arthropods [5,35,36] and two distantly related groups of nematodes [37].

28 Discordant Wolbachia and host phylogenies indicate that many hosts have recently acquired Wolbachia via introgressive and horizontal transfer [38-43]. Wolbachia are primarily transmitted vertically by female hosts, so natural selection favors beneficial effects on host fitness that

31 promote spread [44-47]. Maternal transmission occurs via the host reproductive system, but

32 Wolbachia are also found in host somatic tissues, including nervous, digestive, and metabolic tissues [48-51]. Still, the behavioral and physiological consequences of somatic infections are poorly understood $[19,51]$.

Prior work indicates Wolbachia influence several host behaviors [19,52,53], including sleep [54-56] and temperature preference [20,57,58]. We broadly test for Wolbachia effects on the locomotor activity of Drosophila hosts infected with A-group Wolbachia $(N=11)$, B-group Wolbachia $(N=1)$, and an A- and B-group co-infection $(N=1)$. Our analysis includes two prominent A-group clades that recently spread among Drosophila: wMel-like Wolbachia ( $w$ MelCS, two $w \mathrm{Mel}$ variants, $w$ Yak, $w$ San, and $w$ Tei) and $w$ Ri-like Wolbachia ( $w$ Ri, $w$ Suz, and wAur) $[42,43]$. We find that Wolbachia effects on host activity are common, particularly for

42 wRi-like Wolbachia, a "super-spreader" strain that rapidly spread among Drosophila species in 43 the last $\sim 13,000$ years [42]. 


\section{METHODS}

$48 \quad$ Fly lines

49 We evaluated 13 different Wolbachia-infected host genotypes (Figure 1, Table S1), 50 consisting of nine Drosophila species infected with 14 different A- and B-group Wolbachia that 51 diverged up to 46 million years ago [59]. For two of the host species, D. melanogaster and $D$.

52 simulans, we tested multiple Wolbachia-infected genotypes. This included a D. simulans host co-

53 infected with A-group $w \mathrm{Ha}$ and B-group $w$ No [60-63]. We used tetracycline treatment as

54 previously described [20] to generate uninfected genotypes to pair with each infected genotype,

55 while taking care to avoid detrimental effects of the antibiotic treatment on mitochondrial

56 function [64] (see Supplemental Methods).

Host locomotor activity assays

We reared flies at $25^{\circ} \mathrm{C}$ under a 12-h light:12-h dark cycle (Percival model I-36LL) on a standard food diet [20]. Each day, we collected a batch of female and male virgins for one pair of uninfected and infected genotypes. The four treatment groups (uninfected females, infected females, uninfected males, and infected males) were maintained in isolation until they were 3 to 5 days old. We then measured the locomotor activity of the batch of flies using a 16-chamber flow-through respirometry and data acquisition system (MAVEn, Sable Systems International). The MAVEn has $162.4 \mathrm{ml}$ volume polycarbonate animal chambers and an activity board that uses infrared light (invisible to flies) to monitor animal activity in each chamber, sampled at 1 $\mathrm{Hz}$ (Figure S1). Individual flies were aspirated into a randomly assigned chamber and allowed to adjust to the new environment for 0.5 hours. Activity measurements were then recorded over a 3hour period between the hours of 0900 and 1600.

71 absolute distance sums (ADS). We calculated ADS by first calculating the cumulative sum of the

72 absolute difference between consecutive activity readings, and then calculating the slope of

73 cumulative activity vs. time $[65,66]$. We used mean ADS over the 3-hour period as our estimate

74 of locomotor activity for each fly; however, our analyses were robust regardless of how we

75 quantified activity (see Supplemental Methods). We found that the mean ADS activity data

76 required a transformation for statistical analysis; however, a single data transformation was not

77 suitable for all host species. We used a log transformation of mean ADS for D. simulans, D. 
suzukii, D. auraria, D. mauritiana, and D. sechellia, and a square root transformation for $D$. melanogaster, D. yakuba, D. santomea, and D. teissieri. We present a full statistical analysis of all datasets in Tables S2 and S3, respectively.

We used the log- and square root-transformed mean ADS data as dependent variables in

82 linear models. We included infection status, sex, and an infection-by-sex interaction effect as

83 independent variables, as well as additional independent variables to account for other potential

84 sources of activity variation: randomly assigned animal chamber (1-16), experimental start time,

85 mean water vapor (ppt), mean relative humidity $(\%)$, mean temperature $\left({ }^{\circ} \mathrm{C}\right)$, and mean light intensity (lux) $[65,66]$. We evaluated the significance of individual effects using $F$ tests and type

87 III sum of squares using the "Anova" function in the car R package $[67,68]$.

Wolbachia phylogenomic analysis

We used publicly available Wolbachia genome assemblies [20,42,43,59,69-71], and new

91 Illumina sequencing, to generate a Bayesian phylogram [20] (see Supplemental Methods).

92 Wolbachia effects on host activity were especially common for wRi-like Wolbachia, so we used

93 the phylogram to test whether Wolbachia effects on hosts exhibit phylogenetic signal. First, we

94 treated Wolbachia effects on host locomotor activity as a binary trait and tested for phylogenetic

95 signal using the $D$ statistic [72], implemented in the caper $\mathrm{R}$ package [73]. Second, we treated

96 Wolbachia effects on activity as a continuous trait and tested for phylogenetic signal using

97 Pagel's lambda $(\lambda)$ [74]. Here, we analyzed each sex separately, because we found significant infection-by-sex interaction effects on activity (Tables S2 and S3). For each sex, we extracted the least-square (LS) mean ADS for infected and uninfected flies from the linear models (Tables S2

100 and S3), and used the change in LS mean activity as a continuous character to calculate the maximum likelihood value of Pagel's $\lambda[74,75]$. We used a likelihood ratio test to compare our

102 fitted value of $\lambda$ to a model assuming no phylogenetic signal $(\lambda=0)$ using the "phylosig" 103 function in the R package phytools [76].

\section{RESULTS}

\section{Wolbachia infections modify host locomotor activity}

We assayed the locomotor activity of 3,104 flies (Figure 1). Wolbachia had a significant 
Wolbachia. Interestingly, the direction of Wolbachia effects on host activity varied by genotype and sex (Figure 2). We found a significant Wolbachia infection-by-sex interaction effect for the $w$ MelCS- $D$. melanogaster genotype that increased male activity $(F=4.566, P=0.033$; Table $\mathrm{S} 3)$. We also found a significant infection-by-sex effect for the $w \mathrm{Ri}-D$. simulans genotype, but Wolbachia increased female activity $(F=8.150, P=0.005$; Table $\mathrm{S} 2)$. The two other closely related $w$ Ri-like Wolbachia, $w$ Suz and $w$ Aur, also had significant effects on host activity. The wSuz-D. suzukii genotype had a significant main effect of Wolbachia that reduced host activity $(F=11.311, P<0.001$; Table $\mathrm{S} 2)$, and the $w$ Aur-D. auraria genotype had a significant infection-by-sex interaction that reduced female activity $(F=6.584, P=0.011$; Table S2). The $w$ Ha- $D$. simulans genotype had a significant main effect of Wolbachia that increased host activity $(F=7.764, P=0.006$; Table S2). Lastly, we found the $w$ Ha- $w$ No co-infected $D$. simulans genotype had a significant infection-by-sex interaction effect that reduced male activity $(F=7.076, P=0.008$; Table S2). Because this genotype is co-infected, we do not know the relative contributions of $w \mathrm{Ha}$ and $w$ No to variation in host activity. See the Supplemental Results for a discussion of how other variables contributed to variation in locomotor activity.

\section{Limited evidence for phylogenetic signal}

We estimated a Bayesian phylogram of A- and B-group Wolbachia using 211 single-copy genes of identical length in all Wolbachia genomes, spanning 178,569 bp (Figure 2). We then tested whether closely related Wolbachia have similar effects on host activity. When treating Wolbachia effects on activity as a binary trait, our estimate of $D=0.322$ was low, but not statistically different from a model of $D=1$ assuming phylogenetic randomness $(P=0.101)$ or a model of $D=0$ with strong phylogenetic signal $(P=0.198)$. Simulations of similar phylogenies with an increasing number of Wolbachia strains suggest that at least $N=50$ strains are required to differentiate our estimated value of $D=0.322$ from a model of phylogenetic randomness $(D=$ signal, but many more Wolbachia strains are required to test this hypothesis. Unfortunately, $N=$ 50 strains are not presently available in culture. We also treated Wolbachia changes to host activity as a continuous trait; however, we found that maximum likelihood fitted $\lambda$ values were extremely low, indicative of no phylogenetic signal. $\lambda$ values generated from the LS mean log- 
males $(\lambda<0.001, P=1)$. This was also true when we repeated the analyses for the LS mean square root-transformed $\operatorname{ADS}$ data for females $(\lambda<0.001, P=1)$ and males $(\lambda<0.001, P=1)$.

\section{DISCUSSION}

Our analyses suggest that Wolbachia commonly alter host locomotor activity, which may affect host fitness. Locomotion is a basic host activity underlying many ecologically important behaviors, including foraging, thermoregulation, and mate seeking. In combination with our recent work demonstrating pervasive effects of A- and B-group Wolbachia on host temperature preference [20], we posit that Wolbachia infections may often alter host behavior.

The $w \mathrm{Ri}$-like Wolbachia strains in our study ( $w \mathrm{Ri}, w \mathrm{Suz}$, and $w \mathrm{Aur})$ consistently altered host activity. We found a low, but non-significant $D$ value of 0.322 , suggesting effects on host activity may exhibit phylogenetic signal; although, an excessive number of Wolbachia strains are required to test this hypothesis. Our findings are consistent with prior experiments demonstrating that $w \mathrm{Ri}$ increased female $D$. simulans activity in response to olfactory cues $[77,78]$. We hypothesize that the prominence of $w \mathrm{Ri}$-like Wolbachia effects on host activity relative to other strains may be due to variation in Wolbachia tissue localization [49,52]. wRi occurs at high titer in adult $D$. simulans brains and localizes to specific regions, whereas $w \mathrm{Mel}$ shows a relatively even distribution in D. melanogaster [52]. $w \mathrm{Ri}$ also occurs at higher titer in the ventral nerve cord, which is a major neural circuit center for motor activities such as walking [52,79-81]. Future experiments should compare Wolbachia titer and localization in adult brains for wRi-like variants and strains that do not alter locomotor activity.

We also found considerable variation in the direction and sex-bias of Wolbachia effects on locomotor activity (Figure 2). Wolbachia decreased activity for $w$ Suz, wAur, and the $w$ No$w \mathrm{Ha}$ co-infection, whereas $w \mathrm{MelCS}, w \mathrm{Ri}$, and $w \mathrm{Ha}$ increased activity. These effects were female-biased for $w \mathrm{Ri}$ and $w$ Aur, but male-biased for $w \mathrm{MelCS}$ and $w$ No- $w \mathrm{Ha}$. This variation had no relationship to the Wolbachia phylogeny, because we found no evidence for phylogenetic

\section{Specific Wolbachia effects on host activity may depend on interactions with the host}

168 background. For example, our work and others' suggests that identical $w$ MelCS variants have different effects on D. melanogaster temperature preference depending on the host background [20,57,58]. Host genomes also modify Wolbachia titer [82], Wolbachia maternal transmission 
171 [83], components of host fitness [84-87], and the strength of cytoplasmic incompatibility [88-

$17290]$.

173 Changes to host activity could underlie Wolbachia-induced behaviors that promote

174 infection spread. For example, wMel-infected D. melanogaster have higher field recapture rates

175 than uninfected flies [91], and long distance dispersal of the spider Erigone atra is altered by

176 Rickettsia, an endosymbiont closely related to Wolbachia [92]. Our own work suggests

177 Wolbachia may alter host temperature preference to promote Wolbachia replication within host

178 bodies [20]. Other experiments suggest that $w \mathrm{Mel}$ and $w \mathrm{Ri}$ may influence male mating rate

$179[93,94]$. Alternatively, hosts may be modifying their own behavior as a response to Wolbachia

180 infection. Several studies indicate that $w \mathrm{Mel}$ alters circadian activity and sleep patterns of $D$.

181 melanogaster [52,54-56]. For example, Bi et al. [55] report that wMel increases sleep time,

182 which could represent a host immune response to infection [19]. Ultimately, these effects on host

183 behavior factor into how Wolbachia influence host fitness, which determines the spread and

184 persistence of Wolbachia in host populations [4,95-98]. Because locomotor activity is such a

185 fundamental host behavior, our results suggest Wolbachia may have complex and variable

186 effects on many components of host fitness.

\section{ACKNOWLEDEMENTS}

We thank TBW for lab assistance and WRC for help with bioinformatic analyses. The

190 Cooper lab group and MT provided valuable feedback that improved the manuscript. This work

191 was supported by NIGMS of the NIH under award R35GM124701 to BSC.

\section{DATA AVAILABILITY}

All data and code are available on Dryad (doi:10.5061/dryad.6t1gljwxv; temporary URL:

196 Genome assemblies will be deposited on GenBank upon acceptance. 


\section{FIGURE LEGENDS}

198 Figure 1. Activity of uninfected and infected flies for each sex of each genotype. Activity is

199 measured as mean absolute distance sums (ADS). Significance was evaluated using linear

200 models (Tables S2 and S3).

201

202 Figure 2. (A) Estimated Bayesian phylogram for A- and B-group Wolbachia strains. The

203 divergence estimate for A- and B-groups is superimposed from Meany et al. [59]. All nodes have

204 Bayesian posterior probabilities of 1. (B) Wolbachia effects on host activity scored as a binary

205 trait: Wolbachia significantly altered host activity (black circle) or had no effect (white circle).

206 (C) Wolbachia effects on activity scored as a continuous trait: the change in least-square (LS)

207 mean log-transformed activity (ADS) for each sex. LS means were generated from linear models

208 (Table S2). LS mean square root-transformed ADS data are shown in Figure S3. 


\section{REFERENCES}

210 1. Moran NA, McCutcheon JP, Nakabachi A. 2008 Genomics and evolution of heritable 211 bacterial symbionts. Annual Review of Genetics 42, 165-190.

212 2. McFall-Ngai M et al. 2013 Animals in a bacterial world, a new imperative for the life

213 sciences. Proceedings of the National Academy of Sciences 110, 3229-3236.

214 3. McCutcheon JP, Boyd BM, Dale C. 2019 The life of an insect endosymbiont from the 215 cradle to the grave. Current Biology 29, R485-R495.

4. Hoffmann AA, Turelli M. 1997 Cytoplasmic incompatibility in insects. In Influential passengers: inherited microorganisms and arthropod reproduction (eds SL O'Neill, AA Hoffmann, JH Werren), pp. 42-80. Oxford University Press.

5. Werren JH, Baldo L, Clark ME. 2008 Wolbachia: master manipulators of invertebrate

6. Hurst G, Hurst L, Majerus M. 1997 Cytoplasmic sex-ratio distorters. In Influential passengers: inherited microorganisms and arthropod reproduction (eds SL O'Neill, AA Hoffmann, JH Werren), pp. 125-154. Oxford University Press.

7. Shropshire JD, Leigh B, Bordenstein SR. 2020 Symbiont-mediated cytoplasmic

8. Baumann P. 2005 Biology of bacteriocyte-associated endosymbionts of plant sap-sucking insects. Annual Review of Microbiology 59, 155-189.

9. Douglas AE. 2009 The microbial dimension in insect nutritional ecology. Functional Ecology 23, 38-47.

10. Brumin M, Kontsedalov S, Ghanim M. 2011 Rickettsia influences thermotolerance in the whitefly Bemisia tabaci B biotype. Insect Science 18, 57-66.

11. Mueller UG et al. 2011 Evolution of cold-tolerant fungal symbionts permits winter fungiculture by leafcutter ants at the northern frontier of a tropical ant-fungus symbiosis. Proceedings of the National Academy of Sciences 108, 4053-4056.

12. Hedges LM, Brownlie JC, O’Neill SL, Johnson KN. 2008 Wolbachia and virus protection in insects. Science 322, 702-702.

13. Teixeira L, Ferreira Á, Ashburner M. 2008 The bacterial symbiont Wolbachia induces resistance to RNA viral infections in Drosophila melanogaster. PLoS Biology 6, e1000002. 
15. Oliver KM, Smith AH, Russell JA. 2014 Defensive symbiosis in the real world-advancing ecological studies of heritable, protective bacteria in aphids and beyond. Functional Ecology 28, 341-355.

16. Feldhaar H. 2011 Bacterial symbionts as mediators of ecologically important traits of insect hosts. Ecological Entomology 36, 533-543.

17. Goodacre SL, Martin OY. 2012 Modification of insect and arachnid behaviours by vertically transmitted endosymbionts: infections as drivers of behavioural change and evolutionary novelty. Insects 3, 246-261.

18. Schretter CE, Vielmetter J, Bartos I, Marka Z, Marka S, Argade S, Mazmanian SK. 2018 A gut microbial factor modulates locomotor behaviour in Drosophila. Nature 563, 402-406.

19. Bi J, Wang Y-F. 2020 The effect of the endosymbiont Wolbachia on the behavior of insect hosts. Insect Science 27, 846-858.

20. Hague MT, Caldwell CN, Cooper BS. 2020 Pervasive effects of Wolbachia on host temperature preference. mBio 11.

21. Hosokawa T, Fukatsu T. 2020 Relevance of microbial symbiosis to insect behavior. Current Opinion in Insect Science 39, 91-100.

22. Thomas F, Adamo S, Moore J. 2005 Parasitic manipulation: where are we and where should we go? Behavioural Processes 68, 185-199.

23. Lefevre T, Thomas F. 2008 Behind the scene, something else is pulling the strings: emphasizing parasitic manipulation in vector-borne diseases. Infection, Genetics and Evolution 8, 504-519.

24. Poulin R. 2010 Parasite manipulation of host behavior: an update and frequently asked questions. In Advances in the Study of Behavior, pp. 151-186. Elsevier.

25. van Houte S, Ros VI, van Oers MM. 2013 Walking with insects: molecular mechanisms behind parasitic manipulation of host behaviour. Molecular Ecology 22, 3458-3475.

26. Heil M. 2016 Host manipulation by parasites: cases, patterns, and remaining doubts. Frontiers in Ecology and Evolution 4, 80.

27. Vale PF, Siva-Jothy A, Morrill A, Forbes MR. 2018 The influence of parasites. In Insect behavior: from mechanisms to ecological and evolutionary consequences, pp. 273-291. Oxford University Press Oxford.

28. Weinersmith KL. 2019 What's gotten into you?: A review of recent research on parasitoid manipulation of host behavior. Current Opinion in Insect Science 
29. Louis C, Jourdan M, Cabanac M. 1986 Behavioral fever and therapy in a rickettsia-infected Orthoptera. American Journal of Physiology-Regulatory, Integrative and Comparative Physiology 250, R991-R995.

30. Fedorka KM, Kutch IC, Collins L, Musto E. 2016 Cold temperature preference in bacterially infected Drosophila melanogaster improves survival but is remarkably suboptimal. Journal of Insect Physiology 93, 36-41.

31. Adelman JS, Martin LB. 2009 Vertebrate sickness behaviors: adaptive and integrated neuroendocrine immune responses. Integrative and Comparative Biology 49, 202-214.

32. Opp MR. 2009 Sleeping to fuel the immune system: mammalian sleep and resistance to parasites. BMC Evolutionary Biology 9, 1-3.

33. Kuo T-H, Pike DH, Beizaeipour Z, Williams JA. 2010 Sleep triggered by an immune response in Drosophila is regulated by the circadian clock and requires the NFKB Relish. BMC Neuroscience 11, 1-12.

34. Kuo T-H, Williams JA. 2014 Increased sleep promotes survival during a bacterial infection in Drosophila. Sleep 37, 1077-1086.

35. Zug R, Hammerstein P. 2012 Still a host of hosts for Wolbachia: Analysis of recent data suggests that $40 \%$ of terrestrial arthropod species are infected. PLoS One 7, e38544.

36. Weinert LA, Araujo-Jnr EV, Ahmed MZ, Welch JJ. 2015 The incidence of bacterial endosymbionts in terrestrial arthropods. Proceedings of the Royal Society B: Biological Sciences 282, 20150249.

37. Bandi C, Anderson TJ, Genchi C, Blaxter ML. 1998 Phylogeny of Wolbachia in filarial nematodes. Proceedings of the Royal Society of London. Series B: Biological Sciences $\mathbf{2 6 5}$, 2407-2413.

38. O’Neill SL, Giordano R, Colbert AM, Karr TL, Robertson HM. 1992 16S rRNA phylogenetic analysis of the bacterial endosymbionts associated with cytoplasmic incompatibility in insects. Proceedings of the National Academy of Sciences 89, 26992702.

39. Raychoudhury R, Baldo L, Oliveira DC, Werren JH. 2009 Modes of acquisition of Wolbachia: Horizontal transfer, hybrid introgression, and codivergence in the Nasonia species complex. Evolution 63, 165-183.

40. Conner WR, Blaxter ML, Anfora G, Ometto L, Rota-Stabelli O, Turelli M. 2017 Genome comparisons indicate recent transfer of $w \mathrm{Ri}$-like Wolbachia between sister species Drosophila suzukii and D. subpulchrella. Ecology and Evolution 7, 9391-9404.

41. Gerth M, Bleidorn C. 2017 Comparative genomics provides a timeframe for Wolbachia evolution and exposes a recent biotin synthesis operon transfer. Nature Microbiology $\mathbf{2}$, 16241. 
42. Turelli M et al. 2018 Rapid global spread of wRi-like Wolbachia across multiple Drosophila. Current Biology 28, 963-971.e8.

43. Cooper BS, Vanderpool D, Conner WR, Matute DR, Turelli M. 2019 Wolbachia acquisition by Drosophila yakuba-clade hosts and transfer of incompatibility loci between distantly related Wolbachia. Genetics 212, 1399-1419.

44. Prout T. 1994 Some evolutionary possibilities for a microbe that causes incompatibility in its host. Evolution 48, 909-911.

45. Turelli M. 1994 Evolution of incompatibility-inducing microbes and their hosts. Evolution 48, 1500-1513.

46. Weeks AR, Turelli M, Harcombe WR, Reynolds KT, Hoffmann AA. 2007 From parasite to mutualist: Rapid evolution of Wolbachia in natural populations of Drosophila. PLoS Biology 5, e114.

47. Haygood R, Turelli M. 2009 Evolution of incompatibility-inducing microbes in subdivided host populations. Evolution 63, 432-447.

48. Dobson SL, Bourtzis K, Braig HR, Jones BF, Zhou W, Rousset F, O’Neill SL. 1999 Wolbachia infections are distributed throughout insect somatic and germ line tissues. Insect Biochemistry and Molecular Biology 29, 153-160.

49. Albertson R, Casper-Lindley C, Cao J, Tram U, Sullivan W. 2009 Symmetric and asymmetric mitotic segregation patterns influence Wolbachia distribution in host somatic tissue. Journal of Cell Science 122, 4570-4583.

50. Casper-Lindley C, Kimura S, Saxton DS, Essaw Y, Simpson I, Tan V, Sullivan W. 2011 Rapid fluorescence-based screening for Wolbachia endosymbionts in Drosophila germ line and somatic tissues. Applied Environmental Microbiology 77, 4788-4794.

51. Pietri JE, DeBruhl H, Sullivan W. 2016 The rich somatic life of Wolbachia. MicrobiologyOpen 5, 923-936.

52. Albertson R, Tan V, Leads RR, Reyes M, Sullivan W, Casper-Lindley C. 2013 Mapping Wolbachia distributions in the adult Drosophila brain. Cellular Microbiology 15, 15271544.

53. Fleury F, Vavre F, Ris N, Fouillet P, Bouletreau M. 2000 Physiological cost induced by the maternally-transmitted endosymbiont Wolbachia in the Drosophila parasitoid Leptopilina heterotoma. Parasitology 121, 493-500.

54. Vale PF, Jardine MD. 2015 Sex-specific behavioural symptoms of viral gut infection and Wolbachia in Drosophila melanogaster. Journal of Insect Physiology 82, 28-32.

55. Bi J, Sehgal A, Williams JA, Wang Y-F. 2018 Wolbachia affects sleep behavior in Drosophila melanogaster. Journal of Insect Physiology 107, 81-88. 
56. Morioka E, Oida M, Tsuchida T, Ikeda M. 2018 Nighttime activities and peripheral clock oscillations depend on Wolbachia endosymbionts in flies. Scientific reports 8, 15432.

57. Arnold PA, Levin SC, Stevanovic AL, Johnson KN. 2019 Drosophila melanogaster infected with Wolbachia strain wMelCS prefer cooler temperatures. Ecological Entomology 44, 287-290.

58. Truitt AM, Kapun M, Kaur R, Miller WJ. 2019 Wolbachia modifies thermal preference in Drosophila melanogaster. Environmental Microbiology 21, 3259-3268.

59. Meany MK, Conner WR, Richter SV, Bailey JA, Turelli M, Cooper BS. 2019 Loss of cytoplasmic incompatibility and minimal fecundity effects explain relatively low Wolbachia frequencies in Drosophila mauritiana. Evolution 73, 1278-1295.

60. O'Neill SL, Karr TL. 1990 Bidirectional incompatibility between conspecific populations of Drosophila simulans. Nature 348, 178-180.

61. Mercot H, Llorente B, Jacques M, Atlan A, Montchamp-Moreau C. 1995 Variability within the Seychelles cytoplasmic incompatibility system in Drosophila simulans. Genetics 141, 1015-1023.

62. Rousset F, Solignac M. 1995 Evolution of single and double Wolbachia symbioses during speciation in the Drosophila simulans complex. Proceedings of the National Academy of Sciences 92, 6389-6393.

63. James A, Dean M, McMahon M, Ballard J. 2002 Dynamics of double and single Wolbachia infections in Drosophila simulans from New Caledonia. Heredity 88, 182-189.

64. Ballard J, Melvin R. 2007 Tetracycline treatment influences mitochondrial metabolism and mtDNA density two generations after treatment in Drosophila. Insect Molecular Biology 16, 799-802.

65. Videlier M, Rundle HD, Careau V. 2019 Sex-specific among-individual covariation in locomotor activity and resting metabolic rate in Drosophila melanogaster. The American Naturalist 194, E164-E176.

66. Videlier M, Careau V, Wilson AJ, Rundle HD. 2021 Quantifying selection on standard metabolic rate and body mass in Drosophila melanogaster. Evolution 75, 130-140.

67. Fox J, Weisberg S. 2019 An R Companion to Applied Regression. Third. Thousand Oaks CA: Sage. See https://socialsciences.mcmaster.ca/jfox/Books/Companion/.

68. R Core Team. 2018 R: A language and environment for statistical computing. Vienna, Austria: R Foundation for Statistical Computing. See https://www.R-project.org/.

69. Klasson L et al. 2009 The mosaic genome structure of the Wolbachia wRi strain infecting Drosophila simulans. Proceedings of the National Academy of Sciences 106, 5725-5730. 
70. Ellegaard KM, Klasson L, Näslund K, Bourtzis K, Andersson SG. 2013 Comparative genomics of Wolbachia and the bacterial species concept. PLoS Genetics 9.

71. Siozios S, Cestaro A, Kaur R, Pertot I, Rota-Stabelli O, Anfora G. 2013 Draft genome sequence of the Wolbachia endosymbiont of Drosophila suzukii. Genome announcements 1, e00032-13.

72. Fritz SA, Purvis A. 2010 Selectivity in mammalian extinction risk and threat types: a new measure of phylogenetic signal strength in binary traits. Conservation Biology 24, 10421051.

73. Orme D, Freckleton R, Thomas G, Petzoldt T, Fritz S, Isaac N, Pearse W. 2018 caper: Comparative Analyses of Phylogenetics and Evolution in R. See https://CRAN.Rproject.org/package $=$ caper.

74. Pagel M. 1999 Inferring the historical patterns of biological evolution. Nature 401, 877884.

75. Freckleton RP, Harvey PH, Pagel M. 2002 Phylogenetic analysis and comparative data: a test and review of evidence. The American Naturalist 160, 712-726.

76. Revell LJ. 2012 phytools: an R package for phylogenetic comparative biology (and other things). Methods in Ecology and Evolution 3, 217-223.

77. Peng Y, Nielsen JE, Cunningham JP, McGraw EA. 2008 Wolbachia infection alters olfactory-cued locomotion in Drosophila spp. Applied Environmental Microbiology 74, 3943-3948.

78. Peng Y, Wang Y. 2009 Infection of Wolbachia may improve the olfactory response of Drosophila. Chinese Science Bulletin 54, 1369-1375.

79. Burrows M, Laurent G, Field L. 1988 Proprioceptive inputs to nonspiking local interneurons contribute to local reflexes of a locust hindleg. Journal of Neuroscience $\mathbf{8}$, 3085-3093.

80. Laurent G, Burrows M. 1988 A population of ascending intersegmental interneurones in the locust with mechanosensory inputs from a hind leg. Journal of Comparative Neurology 275, 1-12.

81. Yellman C, Tao H, He B, Hirsh J. 1997 Conserved and sexually dimorphic behavioral responses to biogenic amines in decapitated Drosophila. Proceedings of the national academy of sciences 94, 4131-4136.

82. Funkhouser-Jones LJ, van Opstal EJ, Sharma A, Bordenstein SR. 2018 The maternal effect germline. PLoS Pathogens 3, e190. 
84. Fry A, Palmer M, Rand D. 2004 Variable fitness effects of Wolbachia infection in Drosophila melanogaster. Heredity 93, 379.

85. Dean MD. 2006 A Wolbachia-associated fitness benefit depends on genetic background in Drosophila simulans. Proceedings of the Royal Society of London B: Biological Sciences 273, 1415-1420.

86. Gruntenko NE, Karpova EK, Adonyeva NV, Andreenkova OV, Burdina EV, Ilinsky YY, Bykov RA, Menshanov PN, Rauschenbach IY. 2019 Drosophila female fertility and juvenile hormone metabolism depends on the type of Wolbachia infection. Journal of Experimental Biology 222, jeb195347.

87. Serga SV, Maistrenko OM, Matiytsiv NP, Vaiserman AM, Kozeretska IA. 2021 Effects of Wolbachia infection on fitness-related traits in Drosophila melanogaster. Symbiosis (doi:10.1007/s13199-020-00743-3)

88. Reynolds KT, Hoffmann AA. 2002 Male age, host effects and the weak expression or nonexpression of cytoplasmic incompatibility in Drosophila strains infected by maternally transmitted Wolbachia. Genetics Research 80, 79-87.

89. Cooper BS, Ginsberg PS, Turelli M, Matute DR. 2017 Wolbachia in the Drosophila yakuba complex: Pervasive frequency variation and weak cytoplasmic incompatibility, but no apparent effect on reproductive isolation. Genetics 205, 333-351.

90. Cattel J et al. 2018 Back and forth Wolbachia transfers reveal efficient strains to control spotted wing Drosophila populations. Journal of Applied Ecology 55, 2408-2418.

91. Caragata EP, Real KM, Zalucki MP, McGraw EA. 2011 Wolbachia infection increases recapture rate of field-released Drosophila melanogaster. Symbiosis 54, 55.

92. Goodacre SL, Martin OY, Bonte D, Hutchings L, Woolley C, Ibrahim K, Thomas CG, Hewitt GM. 2009 Microbial modification of host long-distance dispersal capacity. BMC Biology 7, 1-8.

93. De Crespigny FC, Pitt T, Wedell N. 2006 Increased male mating rate in Drosophila is associated with Wolbachia infection. Journal of Evolutionary Biology 19, 1964-1972.

94. De Crespigny FEC, Wedell N. 2007 Mate preferences in Drosophila infected with Wolbachia? Behavioral Ecology and Sociobiology 61, 1229.

95. Hoffmann AA, Turelli M, Harshman LG. 1990 Factors affecting the distribution of cytoplasmic incompatibility in Drosophila simulans. Genetics 126, 933-948.

96. Kriesner P, Hoffmann AA. 2018 Rapid spread of a Wolbachia infection that does not affect host reproduction in Drosophila simulans cage populations. Evolution 72, 1475-1487.

97. Ross PA, Turelli M, Hoffmann AA. 2019 Evolutionary ecology of Wolbachia releases for disease control. Annual Review of Genetics 53. 
bioRxiv preprint doi: https://doi.org/10.1101/2021.02.24.432688; this version posted February 24, 2021. The copyright holder for this preprint

(which was not certified by peer review) is the author/funder, who has granted bioRxiv a license to display the preprint in perpetuity. It is made available under aCC-BY-ND 4.0 International license.

448 98. Kriesner P, Conner WR, Weeks AR, Turelli M, Hoffmann AA. 2016 Persistence of a

449 Wolbachia infection frequency cline in Drosophila melanogaster and the possible role of

450 reproductive dormancy. Evolution 70, 979-997.

451 


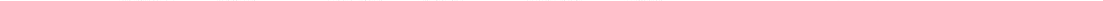




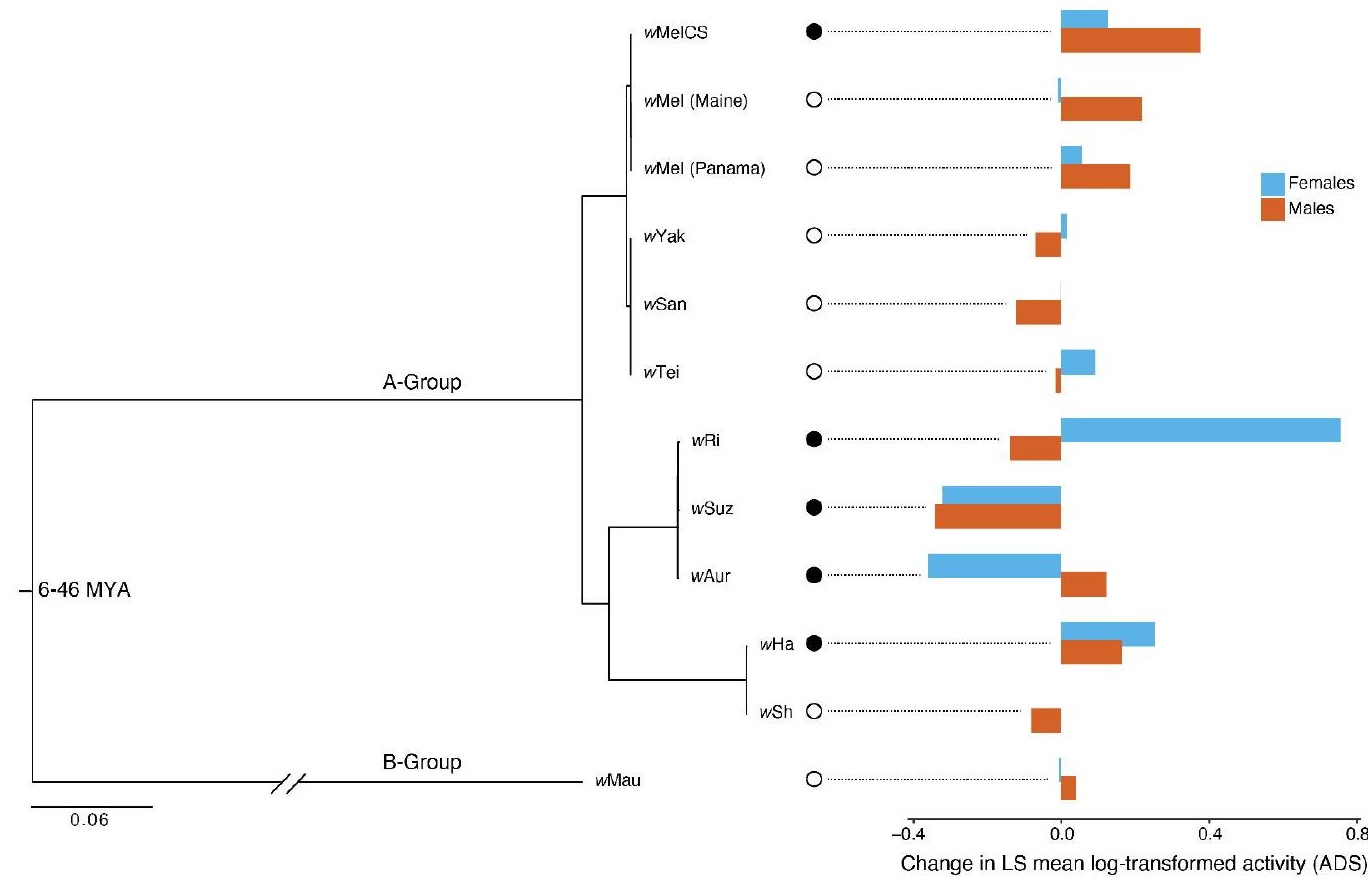

